\title{
Polycyclic Aromatic Nitrogen Heterocycles. Part V: Fluorescence Emission Behavior of Select Tetraaza- and Diazaarenes in Nonelectrolyte Solvents
}

\author{
SHERYL A. TUCKER, WILLIAM E. ACREE, JR., ${ }^{*}$ and CHRISTOPHER UPTON \\ Department of Chemistry, University of North Texas, Denton, Texas 76203-5068, U.S.A. (S.A.T., W.E.A.), and School of \\ Pharmacy and Pharmacology, University of Bath, Claverton Down, Bath, BA2 7AY, England, U.K. (C.U.)
}

\begin{abstract}
Fluorescence emission spectra are reported for tricycloquinazoline, dibenzo[c,f] 2,7$]$ naphthyridine, dibenzo[a,c]phenazine, dibenz[b,h]indeno[1,2,3de][1,6]naphthyridine, and dibenz $[\mathrm{c}, \mathrm{f}]$ indeno[ $1,2,3 \mathrm{ij}]-$ $[2,7]$ naphthyridine dissolved in organic nonelectrolyte solvents of varying polarity and acidity. Results of these experiments were used to screen PANHs for potential probe character. The effect of nitromethane as a selective quenching agent on both the unprotonated and protonated PANHs was also examined. Nitromethane was found to quench fluorescence emission of dibenzo[c,f]|2,7]naphthyridine. Emission intensities of the remaining four PANHs, and of the three protonated PANHs for which emission spectra could be obtained, remained essentially constant and were not affected by nitromethane.

Index Headings: Fluorescence; Spectroscopic techniques; UV-visible spectroscopy.
\end{abstract}

\section{INTRODUCTION}

This paper continues a systematic, experimental investigation $^{1-5}$ of the effect that solvent polarity and acidity has on the photophysical properties of polycyclic aromatic nitrogen heterocycles (PANHs). Pertinent photophysical processes of PANHs dissolved in fluid solution are summarized below:

Unprotonated:

$$
\begin{aligned}
& \text { PANH }+h \nu_{0} \rightarrow \text { PANH }^{*} \\
& \text { (absorption/fluorescence excitation) } \\
& \text { PANH }^{*} \rightarrow \text { PANH }+h \nu_{1}
\end{aligned}
$$$$
\text { (fluorescence emission) }
$$

\section{Protonated:}

$$
\begin{gathered}
\text { PANH-H }^{+}+h \nu_{0} \rightarrow \text { PANH-H } \\
\text { (absorption/fluorescence excitation) } \\
\text { PANH-H }{ }^{+*} \rightarrow \text { PANH-H } \\
\text { PA }^{+}+h \nu_{2}
\end{gathered}
$$

(fluorescence emission)

The emission spectrum of the neutral PANH monomer generally consists of several major bands labeled I, II, etc., in progressive order. Previous measurements revealed that 12-azabenzo[a]pyrene, phenanthro[2,3h]isoquinoline, phenanthro[3,2h]isoquinoline, 1-azabenz[a]anthracene, 2-azabenz[a] anthracene, and 9-azabenz[a] anthracene exhibited modest selective emission

\footnotetext{
Received 25 September 1992.

* Author to whom correspondence should be sent.
}

intensity reduction of band I relative to band II (or band III) in polar solvents. The ratio of emission intensities serves as a quantitative measure of solvent polarity and structure. Interestingly, not all PANHs behave in this fashion. Various emission intensity ratios of 1-azapyrene, 2-azapyrene, 4-azapyrene, 4-azachrysene, benzo[lmn]$[3,8]$ phenanthroline, and benzo[4,10]anthra[ $1,9,8 \mathrm{cdef}]-$ cinnoline remained constant, irrespective of solvent polarity. Fluorescence emission spectra of benzo[a]phenazine, acenaphtho[1,2]pyridine, indeno[1,2,3ij]isoquinoline, and indeno[ $1,2,3 \mathrm{ij}][2,7]$ naphthyridine showed very little structured emission. Depending upon the solute and solvent combination being studied, either one unsymmetrical band or two poorly resolved peaks were observed.

Protonation of the nitrogen lone electron pair by a hydrogen ion results in loss of emission fine structure accompanied by a sizeable red shift in emission wavelength(s) (i.e., $\nu_{1}>\nu_{2}$ ). Degree of protonation is reflected by both the solvent acidity and PANH basicity. For example, 2,2,2-trifluoroethanol completely protonated 12 azabenzo[a]pyrene, 4-azapyrene, phenanthro[2,3h]isoquinoline, phenanthro[3,2h]isoquinoline, and phenanthro $[9,10 \mathrm{~g}]$ isoquinoline. The original three-band emission spectra of 12-azabenzo[a]pyrene at $\sim 395-450$ $\mathrm{nm}$ disappeared and was replaced by a fairly broad, single band red-shifted by over $60 \mathrm{~nm}$ into the $470-560 \mathrm{~nm}$ spectral region. The spectra remained unchanged whenever a small perchloric acid drop was added to the solvent. As expected, the protonation was completely reversible. Addition of sodium hydroxide completely restored the original PANH spectrum, though a slight loss in emission intensity was observed. 1-Azapyrene and 2 -azapyrene, on the other hand, were only partially protonated by trifluoroethanol, as indicated by the presence of both the neutral and protonated species in the emission spectra. Protonation was complete upon addition of perchloric acid, as documented by the disappearance of the first emission band. ${ }^{3}$

Concurrent with the polarity and protonation studies, we also investigated the ability of nitromethane and 1,2,4trimethoxybenzene to act as selective quenching agents to distinguish between alternant and nonalternant PANH molecules. ${ }^{1,5}$ This particular classification scheme is analogous to the one used for polycyclic aromatic hydrocarbons (PAHs). In alternant PAHs, every alternant carbon atom in the aromatic ring system(s) can be "starred." 


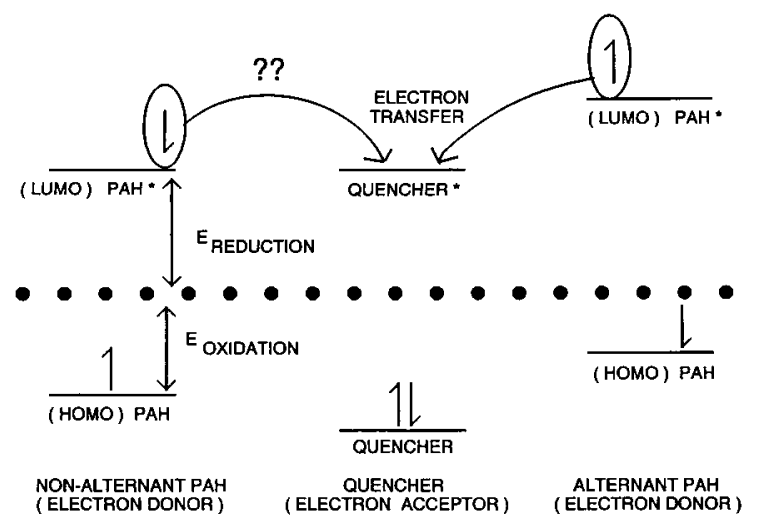

FIG. 1. Simplified molecular orbital diagram indicating favorable conditions for electron transfer between an electron donor alternant PAH and an electron acceptor quenching agent. The quencher's LUMO and nonalternant PAH's LUMO were placed at energies so as to discourage electron transfer. The dotted line represents the potential of a reference electrode. Nitromethane quenches only fluorescence emission of alternant PAHs. Intensities of nonalternant PAHs remain unchanged for the most part; however, a few exceptions are known. More detailed molecular orbital diagrams are given elsewhere. ${ }^{13}$

Nonalternant PAHs, on the other hand, would have at least one pair of adjacent starred atoms. ${ }^{6,7}$ Aromatic carbon atoms and perimeter $-\mathrm{N}=$ atoms are treated identically. Blümer and Zander ${ }^{8}$ recommended that nitromethane and/or nitrobenzene could be added to an aqueous-acetonitrile (20:80 percent by volume) binary mobile phase to selectively suppress fluorescence signals of alternant PAHs. Emission intensities of nonalternant PAHs would remain unchanged. Published studies ${ }^{9-14}$ involving over $63 \mathrm{PAHs}$ have identified dibenzo[hi,wx]heptacene, benzo[k]fluoranthene, fluoranthene, and naphtho[2,3b]fluoranthene as among the few exceptions to the so-called nitromethane selective quenching rule in the PAH6 benzenoid, fluorenoid, fluoranthenoid, and "methylene-bridged" cyclopenta-PAH subclasses.

Quenching behavioral differences between alternant and nonalternant PAHs can be rationalized in terms of processes originating from the vibrationally relaxed first electronic excited singlet state, ${ }^{1} \mathrm{PAH}^{*}$. Deactivation from the ${ }^{1} \mathrm{PAH}^{*}$ state is governed by the competition between radiative and nonradiative processes. Rate constants of fluorescence decay, $k_{\text {fuores }}$, for PAH fluorophores are generally insensitive to molecular environment. Efficiencies of nonradiative processes, on the other hand, depend to a large extent upon external perturbations resulting from interactions involving PAH solutes with solvent/quenching molecules. Three possible mechanisms include reduction of fluorescence emission intensities through: (1) intersystem crossing processes involving external "heavy atom" quenchers:

$$
{ }^{1} \mathrm{PAH}^{*}+{ }^{1} \text { Quencher } \rightarrow{ }^{3} \mathrm{PAH}^{*}+{ }^{1} \text { Quencher; }
$$

(2) intermolecular transfer (or partial transfer; $\delta+/ \delta-$ ) of a single electron to form free ions (or charge transfer complex):

$$
\begin{aligned}
{ }^{1} \mathrm{PAH}^{*}+{ }^{1} \text { Quencher } & \rightleftharpoons\left(\mathrm{PAH}^{ \pm}-- \text {Quencher }^{\mp}\right) \\
& \rightarrow{ }^{2} \mathrm{PAH}^{ \pm}+{ }^{2} \mathrm{Quencher}^{\mp} ;
\end{aligned}
$$

and (3) intermolecular electronic energy transfer:

$$
{ }^{1} \mathrm{PAH}^{*}+{ }^{1} \text { Quencher } \rightarrow{ }^{1} \mathrm{PAH}+{ }^{3} \text { Quencher* }
$$

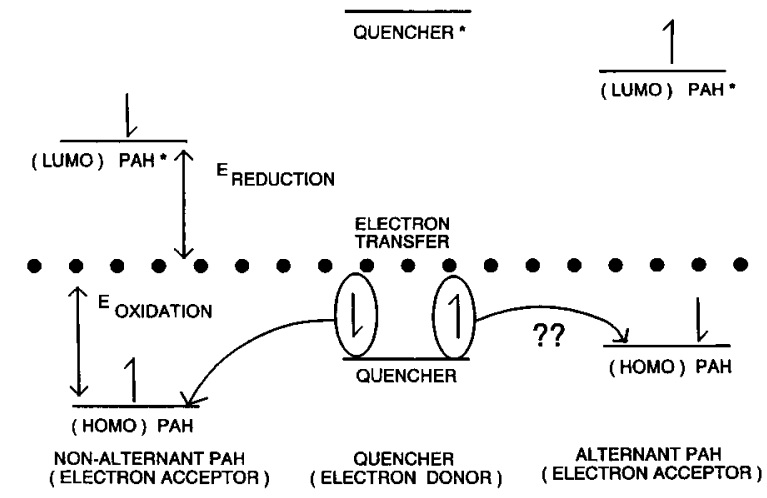

FIG. 2. Simplified molecular orbital diagram indicating favorable conditions for electron transfer between an electron acceptor nonalternant $\mathrm{PAH}$ and an electron donor quenching agent, such as 1,2,4-trimethoxybenzene. More detailed molecular orbital diagrams are given elsewhere. ${ }^{13}$

with the quenching agent promoted from a ground singlet state to an excited triplet state, requiring Energy $\left({ }^{1} \mathrm{PAH}^{*}\right)>$ Energy $\left({ }^{3}\right.$ Quencher $\left.{ }^{*}\right)$.

Of the aforementioned mechanisms, only the second could discriminate between alternant and nonalternant PAHs. Breymann et al. ${ }^{13}$ attributed nitromethane's selectivity to an electron/charge transfer reaction. As argued by the authors, reduction potentials of nonalternant PAHs are generally $0.4 \mathrm{eV}$ more positive than those of alternant PAHs. Quantum mechanical computations show the highest occupied molecular orbital (HOMO) and lowest unoccupied molecular orbital (LUMO) energies of nonalternant PAHs to be lowered against those of alternant PAHs of equal HOMO-LUMO separation. For the electron transfer reaction

$$
{ }^{1} \mathrm{PAH}^{*}+{ }^{1} \text { Quencher } \rightarrow{ }^{2} \mathrm{PAH}^{+}+{ }^{2} \mathrm{Quencher}^{-}
$$

the change in free energy is expected to be more negative in the case of alternant PAHs (see Fig. 1). Assuming that one is in a Marcus-type region, a more favorable electron transfer process results in larger quenching rate constants which, if comparable to (or larger than) $k_{\text {fluores }}$, would cause a significant reduction in $\mathrm{PAH}$ emission intensity. Slow electron transfer reactions (i.e., $k_{\text {quench }} \ll$ $k_{\text {fuores }}$ ) are not expected to affect fluorescence intensities, as the photon is emitted long before electron transfer from the PAH donor to the quencher acceptor can occur. A similar argument could be made for electron donor quenching agents. Here, the quenching agent is more likely to affect fluorescence emission of the nonalternant PAHs (see Fig. 2).

Experimental results from our preliminary quenching studies involving polycyclic aromatic nitrogen heterocyclic solutes showed that nitromethane quenched fluorescence emission of approximately two-thirds of the seventeen alternant PAHNs considered. Corrected emission intensities of the three nonalternant PANHs-acenaphtho[1,2b]pyridine, indeno[ $[1,2,3 \mathrm{ij}]$ isoquinoline, and indeno[ $1,2,3 \mathrm{ij}][2,7]$ naphthyridine - and of all twenty-one protonated PANHs studied thus far remained essentially constant upon nitromethane addition. To explain these observations we proposed the molecular orbital diagram depicted in Fig. 3. A fairly broad reduction potential range for the PANH solutes was assumed. Depending 


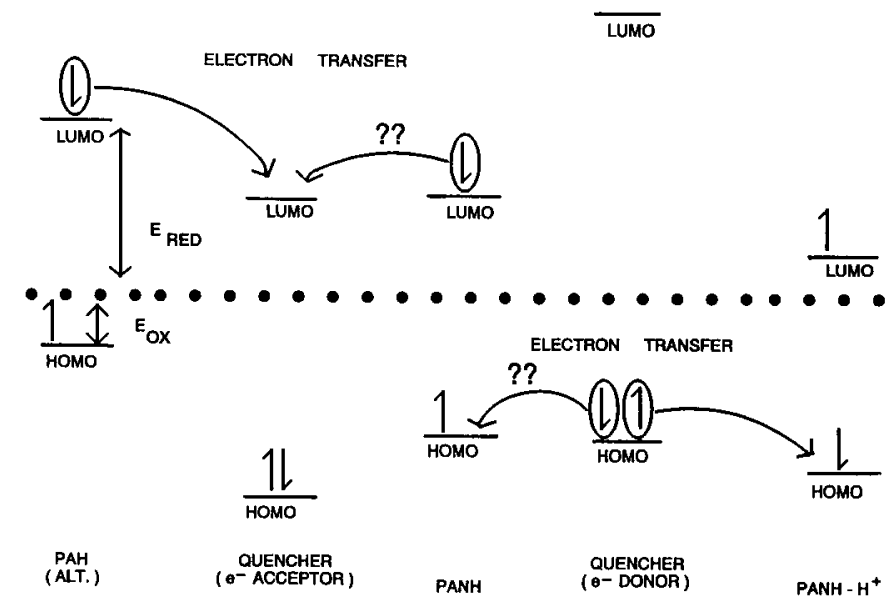

FIG. 3. Plausible molecular orbital diagram to explain observed quenching of unprotonated and protonated PANH fluorescence emission by nitromethane (electron acceptor) and 1,2,4-trimethoxybenzene (electron donor)

upon the molecule under examination, the PANH LUMO fell slightly above (or slightly below) the electron acceptor's LUMO, thereby encouraging (or discouraging) electron transfer. Protonation of the nitrogen lone electron pair by a hydrogen ion caused a decrease in the HOMOLUMO energy separation, as evidenced by a sizeable red shift in the fluorescence emission wavelength(s). Of the five possible ways to decrease the HOMO-LUMO energy gap, the nitromethane quenching data on the protonated PANHs suggest that the LUMO must be moved to give a more positive reduction potential so as to discourage electron transfer from the PANH to nitromethane, which functions as an electron acceptor. The relative location of the unexcited PANH's and PANH-H's highest occupied molecular orbital was ascertained by studying the solute's fluorescence behavior in the presence of an electron donor quenching agent, such as 1,2,4-trimethoxybenzene. Measurements on benz[de]isoquino[1,8gh]quinoline, benzo[a]phenazine, 12-azabenzo[a]pyrene, and diphenanthro $\left[9,10,1 \mathrm{def} ; 1^{\prime}, 1^{\prime}, 9^{\prime}\right.$ hij] phthalazine revealed that trimethoxybenzene quenched the fluorescence emission of the protonated forms of these four PANH solutes. Only benz[de]isoquino[1,8gh]quinoline and 12 -azabenzo[a]pyrene were significantly quenched as neutral PANH molecules.

To expand our existing PANH spectral data file, and to gather additional experimental evidence to support our proposed molecular orbital-quenching mechanism, we have measured the fluorescence behavior of tricycloquinazoline, dibenzo[c,f][2,7]naphthyridine, dibenzo[a,c]phenazine, dibenz [b,h]indeno[1,2,3de][1,6]naphthyridine, and dibenz $[\mathrm{c}, \mathrm{f}]$ indeno $[1,2,3 \mathrm{ij}][2,7]$ naphthyridine (see Fig. 4 for molecular structures) dissolved in organic nonelectrolyte solvents of varying polarity and acidity. Tricycloquinazoline is considered a tetraazaarene for purposes of this study. X-ray crystallographic data ${ }^{15}$ show tricycloquinazoline to be planar to within $\pm 0.05 \AA$, and the molecule possesses considerable aromaticity around the periphery but with a large degree of bond fixation and nonaromaticity in the three inner rings resulting from the three sigma-single bonds originating from the "bridge-head" nitrogen hetero-atom. ${ }^{16}$ Also included in
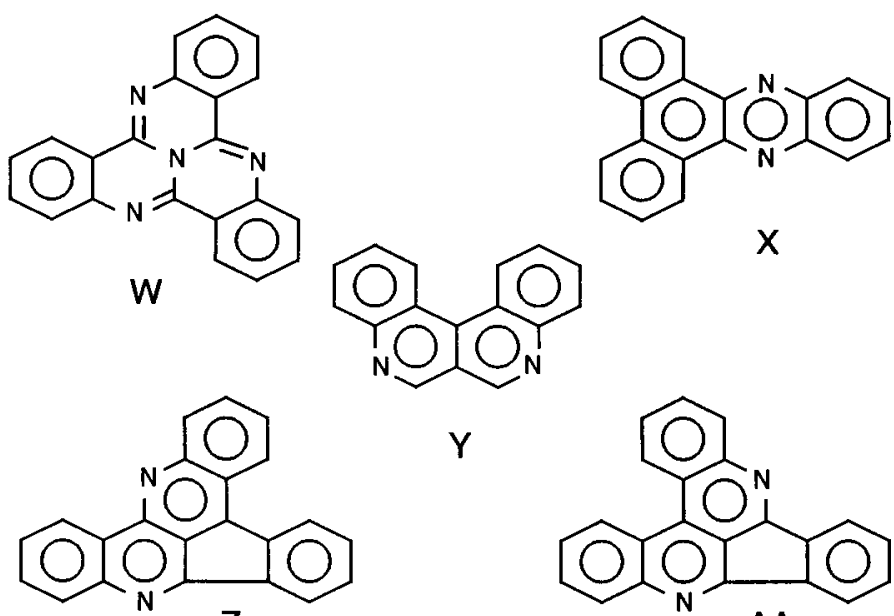

Z

AA

FIG. 4. Molecular structures of PANH solutes: (W) tricycloquinazoline; (X) dibenzo[a,c]phenazine; (Y) dibenzo[c,f][2,7]naphthyridine; $(\mathbf{Z})$ dibenz $[\mathrm{b}, \mathrm{h}]$ indeno[ $[1,2,3 \mathrm{de}][1,6]$ naphthyridine; and (AA) dibenz[c,f]indeno[1,2,3ij][2,7]naphthyridine. To facilitate indexing, Chemical Abstract's nomenclature is used for the chemical names.

this paper is a spectrophotometric estimation of the "apparent" acid dissociation constant of the benzo[a]phenazonium ion in mixed aqueous-acetonitrile solvent ( $30 \%$ acetonitrile by weight) and the effect that nitromethane has on the fluorescence emission intensities of the five neutral and protonated PANH solutes.

\section{MATERIALS AND METHODS}

The five PANH solutes were synthesized and purified by procedures described in the chemical literature. ${ }^{17-21}$ Stock solutions were prepared by dissolving the solutes in dichloromethane. Small aliquots of the stock solutions were transferred into test tubes, allowed to evaporate, and diluted with the solvent of interest. Final solute concentrations were sufficiently dilute to minimize innerfiltering artifacts. Solvents were of HPLC, spectro-quality or AR grade, purchased commercially from either Aldrich or Fisher Scientific, and the resulting solutions were optically dilute (absorbances $\mathrm{cm}^{-1}<0.01$ ) at all wavelengths, except for the nitromethane quenching studies.

Absorption spectra were recorded on a Bausch and Lomb Spectronic 2000 and a Hewlett-Packard $8450 \mathrm{~A}$ photodiode array spectrophotometer in the usual manner. The fluorescence spectra were run on a Shimadzu RF-5000U spectrofluorometer with the detector set at high sensitivity. Solutions were excited at $390 \mathrm{~nm}$ (tricycloquinazoline), $300 \mathrm{~nm}$ (dibenzo[c,f][2,7]naphthyridine), $365 \mathrm{~nm}$ (dibenzo[a,c]phenazine), $400 \mathrm{~nm}$ (dibenz $[\mathrm{b}, \mathrm{h}]$ indeno[ $1,2,3 \mathrm{de}][1,6]$ naphthyridine), and $300 \mathrm{~nm}$ (dibenz[c,f]indeno[1,2,3ij] $[2,7]$ naphthyridine) in a quartz $1-\mathrm{cm}^{2}$ cuvette. Fluorescence data were accumulated at $19^{\circ} \mathrm{C}$, ambient room temperature, with excitation and emission slit-width settings of $15 \mathrm{~nm}$ and $3 \mathrm{~nm}$, respectively. The PANH fluorescence spectra, depicted in Figs. 5 and 6 , represent a single scan which was then solvent blank corrected and verified by repetitive measurements.

Emission intensities associated with the quenching study were corrected for primary inner-filtering artifacts arising from the absorption of excitation radiation. Many 


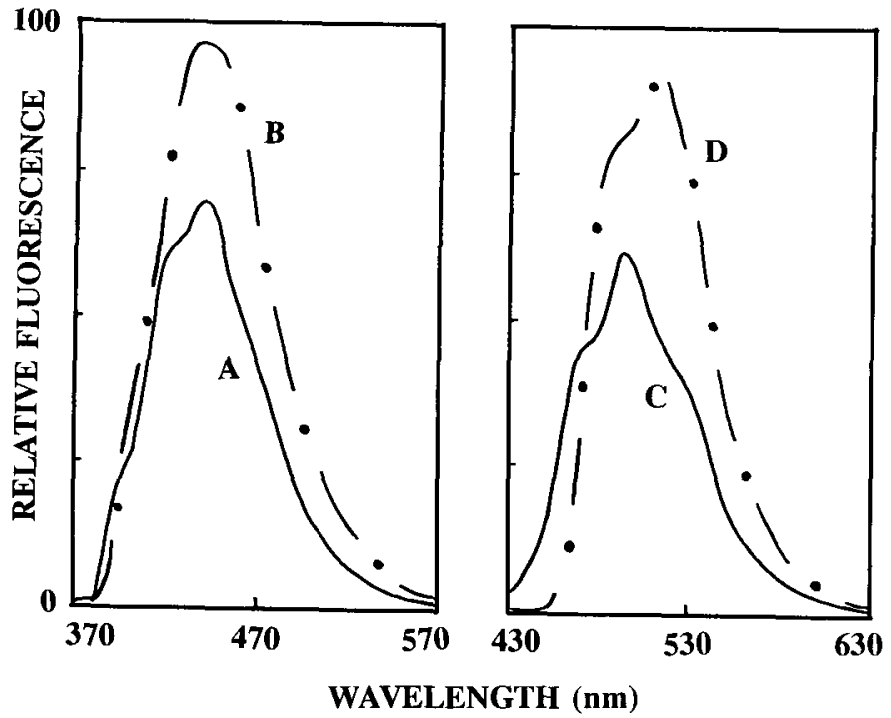

Fig. 5. Fluorescence emission spectra of dibenzo[c,f]indeno$[1,2,3 \mathrm{ij}][2,7]$ naphthyridine dissolved in $[A(\longrightarrow)] n$-hexadecane and in $[B(-)]$ dimethyl sulfoxide, and of dibenz $[\mathrm{b}, \mathrm{h}]$ indeno $[1,2,3 \mathrm{de}][1,6]-$ naphthyridine dissolved in $[C(-)] n$-hexadecane and in $[D(-)]$ dimethyl sulfoxide.

of the PANHs have excitation wavelengths in the 300 $320 \mathrm{~nm}$ spectral region, and a few drops of nitromethane gave solutions having appreciable absorbances. Mathematical expressions, computational procedures, and interrogation zone dimensions are given elsewhere. ${ }^{1,10,22} \mathrm{Ev}$ ery effort was made to work at solution absorbances below $A \mathrm{~cm}^{-1} \leq 0.95\left(f_{\text {prim }} \leq 3.00\right)$ where the inner-filtering correction equation is valid. Secondary inner-filtering corrections were not necessary in the present study since nitromethane is "optically transparent" in most of these PANHs' emission ranges.

"Apparent" acid dissociation constants of two protonated PANHs dissolved in a mixed aqueous-acetonitrile

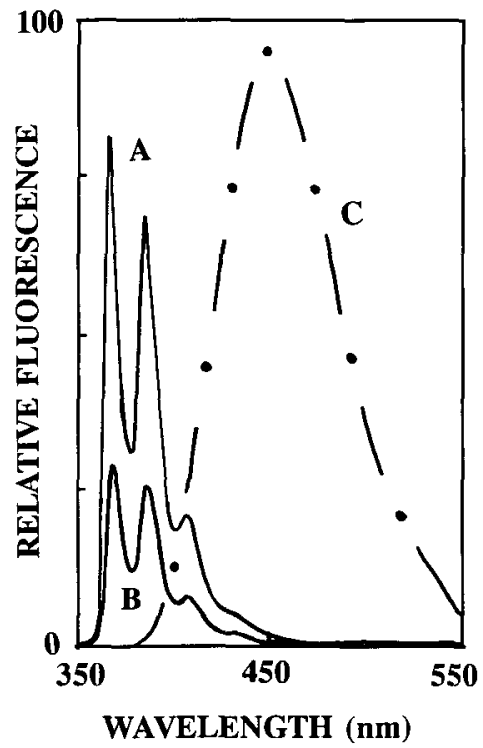

FIG. 6. Fluorescence emission spectra of dibenzo[c,f][2,7]naphthyridine dissolved in $[A(-)]$ acetonitrile; $[B(-)]$ acetonitrile with one pasteur pipet drop of nitromethane; and [C $(-)$ ] acetonitrile-perchloric acid protonating solvent. In acetonitrile, emission bands occur at about $366,384,405$, and $430 \mathrm{~nm}$.

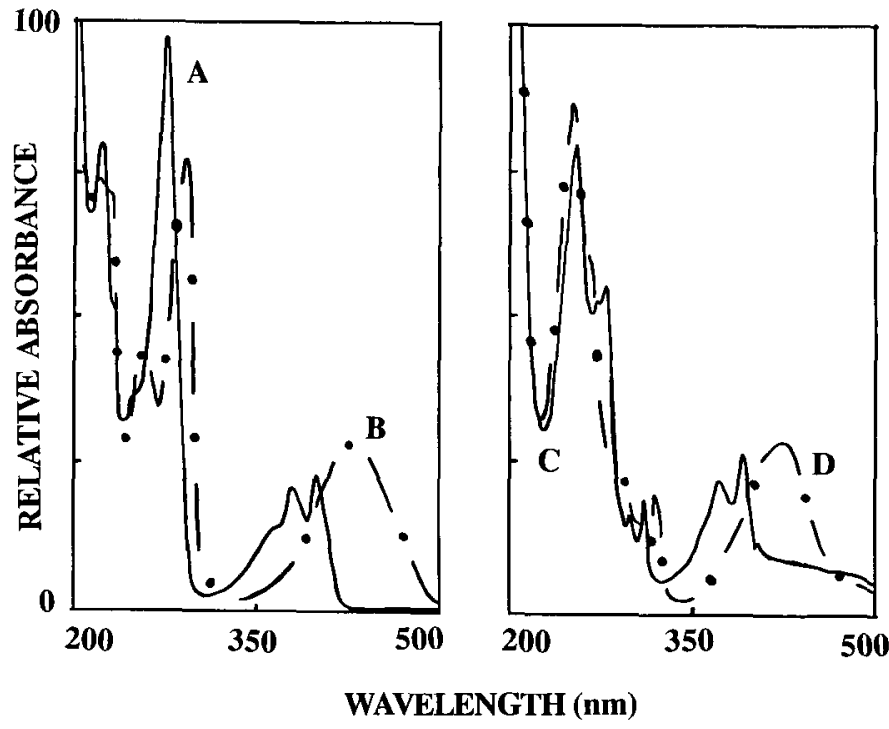

FIG. 7. Absorbance spectra of unprotonated $[A(-)]$ and protonated [ $B(-)]$ benzo[a]phenazine, and of unprotonated $[C(-)]$ and protonated $[D(--)]$ dibenzo[a,c]phenazine.

solvent ( $30 \%$ acetonitrile by weight) were estimated from solution absorbances as a function of $\mathrm{pH}$ while holding the stoichiometric moles of PANH constant. Dissolved PANHs were initially protonated by addition of a few drops of concentrated perchloric acid. Absorbances were monitored at 427 and $425 \mathrm{~nm}$, which are the wavelengths corresponding to the dibenzo[a,c]phenazonium and benzo[a]phenazonium ions, respectively. (See Fig. 7 for representative absorption spectra.) All absorbances were corrected for dilution resulting from addition of a sodium hydroxide solution required to change the $\mathrm{pH}$. Nonaqueous $\mathrm{pH}$-values were measured indirectly as a millivolt reading with a Model 501 Orion Research digital ionalyzer equipped with a standard combination glass electrode. Millivolt readings were converted to $\mathrm{pH}$-values via

$$
\mathrm{pH}_{x}=\mathrm{pH}_{s}-\left(E_{x}-E_{s}\right) / S
$$

where $\mathrm{pH}_{x}$ and $\mathrm{pH}_{s}$ refer to the $\mathrm{pH}$ of the sample $(x)$ and standard (s), respectively, $E_{x}$ and $E_{s}$ are the millivolt values of the sample and standard as read by the $\mathrm{pH}$ meter, and $S$ is the Nernstian slope, which is normally $57.18 \mathrm{mV} / \mathrm{pH}$ unit at $15^{\circ} \mathrm{C} .{ }^{23-25} \mathrm{~A} 0.05$ molal solution of potassium hydrogen phthalate in the aqueous-acetonitrile solvent mixture served as the reference standard buffer, $\mathrm{pH}_{s}=5.030$ at $15^{\circ} \mathrm{C},{ }^{26}$ for all of the $\mathrm{pH}$ measurements.

\section{RESULTS AND DISCUSSION}

Fluorescence emission spectra were recorded for tricycloquinazoline, dibenzo[c,f] [2,7]naphthyridine, dibenzo[a,c]phenazine, dibenz[b,h]indeno[1,2,3de][1,6]naphthyridine, and dibenz $[c, f]$ indeno[1,2,3ij] [2,7]naphthyridine dissolved in cyclohexane, 2,2,4-trimethylpentane, $n$-hexadecane, carbon tetrachloride, dibutyl ether, benzene, 2-propanol, chloroform, butyl acetate, methanol, dichloromethane, acetonitrile, N,N-dimethylformamide, and dimethyl sulfoxide. Representative spectra are depicted in Figs. 5 and 6. The nonelectrolyte solvent set was judiciously selected so as to encompass the entire 
range of solvent polarity, from the nonpolar saturated hydrocarbons to the moderately polar butyl acetate and dichloromethane to the very polar dimethyl sulfoxide. Alcoholic solvents such as methanol and 2-propanol are included to enable assessment of possible solvent acidity probe character.

Examination of the spectral data reveals that tricycloquinazoline had four observable emission bands in the 420-650 nm spectral region, situated at approximately $456,477,512$, and $547 \mathrm{~nm}$. Emission bands near 602 and $650 \mathrm{~nm}$, which appear in the published corrected spectra reported by Cundall and co-workers, ${ }^{27}$ were not observed. At the present time we are unable to explain why these latter two bands are missing; however, we suspect that the reason may be related to differences in chemical purities, concentrations, and/or spectrofluorometers used. We were able to reproduce the authors' absorption spectra. Calculated emission intensity ratios for both tricycloquinazoline and dibenzo[c,f][2,7]naphthyridine varied only slightly in the fourteen solvents considered. Dibenzo[a,c]phenazine and the two nonalternant PANHs (dibenz [b,h]indeno[1,2,3de][1,6]naphthyridine and dibenz $[c, f]$ indeno[1,2,3ij][2,7]naphthyridine; see Fig. 5) showed very little emission fine structure. All five PANHs examined for possible solvent polarity probe behavior in the present study are thus classified as nonprobe molecules.

Polycyclic aromatic nitrogen hetero-atoms are susceptible to protonation, particularly in the more acidic environments. In the case of tricycloquinazoline, dibenzo[c,f][2,7]naphthyridine, and dibenz[c,f]indeno$[1,2,3 \mathrm{ij}][2,7]$ naphthyridine, protonation of the nitrogen lone electron pair by a hydrogen ion resulted in the loss of emission fine structure accompanied by a sizeable red shift in emission wavelength(s). As shown in Fig. 3, protonation must reduce the HOMO and LUMO separation since the emission wavelength is inversely proportional to the energy of the emitted photon. Interestingly, no emission signal was obtained for dibenzo[a,c]phenazine and dibenz $[\mathrm{b}, \mathrm{h}]$ indeno[1,2,3de][1,6]naphthyridine dissolved in an acetonitrile-perchloric acid solution. Protonation did occur, however, as the solution turned visually yellow in color when the acid was added. Figure 7 shows the changes in the UV-visible absorption spectra. Loss of emission signal likely resulted from a shift in the excitation wavelength and/or a decreased quantum efficiency, combined with inner-filtering artifacts caused by absorption of significant quantities of excitation/ emission radiation by the protonated PANH. Original fluorescence spectra were restored by addition of sodium hydroxide, indicating that protonation was reversible.

"Apparent" acid dissociation constants were estimated for two of the three PANHs (dibenzo[a,c]phenazine and benzo[a]phenazine ${ }^{5}$ ) which gave yellow solutions upon addition of perchloric acid. A suitable wavelength could not be found in the case of dibenz[b,h]indeno$[1,2,3 \mathrm{de}][1,6]$ naphthyridine. Assuming that the yellow color corresponds to the protonated PANH:

$$
\begin{aligned}
\mathrm{PANH}+\mathrm{H}^{+} & \rightleftharpoons \mathrm{PANH}-\mathrm{H}^{+} \\
\mathrm{K}_{a} & =\left[\mathrm{PANH}^{+} \mathrm{H}^{+}\right] /[\mathrm{PANH}]\left[\mathrm{H}^{+}\right],
\end{aligned}
$$

then $K_{a}$ can be experimentally obtained by measuring the solution absorbance as a function of $\mathrm{pH}$ at a wave- length where only $\mathrm{PANH}-\mathrm{H}^{+}$absorbs radiation. The BeerLambert law states that absorbance is directly proportional to the light-absorbing species, i.e., $A=$ constant $\left[\mathrm{PANH}-\mathrm{H}^{+}\right]$. At one-half the maximum absorbance (corrected for dilution) the molar concentrations of PANH$\mathrm{H}^{+}$and PANH are identical, and the hydrogen ion concentration equals $\mathrm{K}_{a}$ (alternatively expressed as $\mathrm{pH}=$ $\mathrm{pK}_{a}$ ). The experimental procedure becomes more involved if both the protonated and unprotonated forms absorb radiation. Experimental acid dissociation constants were $\mathrm{pK}_{a} \approx 0.50 \pm 0.15$ and $\mathrm{pK}_{a} \approx 1.35 \pm 0.15$ for dibenzo[a,c]phenazine and benzo[a]phenazine, respectively, dissolved in mixed aqueous-acetonitrile solvent ( $30 \%$ acetonitrile by weight). These values are in line with tabulated values of Markgraf and $\mathrm{Katt}^{28}$ for phenazine $\left(\mathrm{pK}_{a}=1.23\right)$ and dibenzo[a,c]phenazine $\left(\mathrm{pK}_{a}\right.$ $=0.30$ ) dissolved in acetic anhydride. The authors attributed the difficulty in protonating dibenzo[a,c]phenazine to steric inhibition of solvation of the PANH-H ${ }^{+}$ion caused by the two added benzo-rings. Similar effects have been observed in the reactivity of benzo[a]phenazine, in that the nitrogen atom nearest to the added benzo-ring is least reactive. ${ }^{29}$

As part of the present study, we also examined the effect that nitromethane has on PANH and $\mathrm{PANH}-\mathrm{H}^{+}$ fluorescence emission. Of the five PANHs studied, nitromethane was found to quench emission of only dibenzo[c,f] $[2,7]$ naphthyridine. Emission intensities of the remaining four PANHs, and of the three protonated PANHs for which an emission spectra could be obtained, remained essentially constant and were unaffected by nitromethane. These measurements provide further experimental evidence to support our proposed molecular orbital-quenching mechanism depicted in Fig. 3. The fact that nitromethane quenched only one of the two alternant PANHs (dibenzo[c,f][2,7]naphthyridine and dibenzo[a,c]phenazine) studied suggests that the reduction potential range for PANH solutes is fairly broad. Depending upon the actual molecule under examination, the PANH LUMO fell slightly above (or slightly below) the electron acceptor's LUMO, thereby encouraging (or discouraging) electron transfer. Fluorescence emission of protonated PANHs is not quenched by nitromethane, and to date we have measurements on twenty-four different PANH$\mathrm{H}^{+} \mathrm{s}$ to support this claim.

\section{ACKNOWLEDGMENTS}

This work is supported in part by grants from the National Science Foundation (Grant No. CTS-8922485), by the University of North Texas Research Council, and by a National Science Foundation Doctoral Research Fellowship awarded to S. A. Tucker. The authors also thank H. Bates for making several of the preliminary fluorescence measurements.

1. S. A. Tucker, W. E. Acree, Jr., M. J. Tanga, S. Tokita, K. Hiruta, and H. Langhals, Appl. Spectrosc. 46, 229 (1992).

2. S. A. Tucker, W. E. Acree, Jr., M. J. Tanga, M. Zander, J. C. Fetzer, S. Tokita, K. Hiruta, K. Kitahara, and H. Nishi, Appl. Spectrosc. 45, 1188 (1991).

3. S. A. Tucker, W. E. Acree, Jr., and M. J. Tanga, Appl. Spectrosc. 45, 911 (1991).

4. S. A. Tucker, W. E. Acree, Jr., and M. J. Tanga, Appl. Spectrosc. 45, 57 (1991).

5. S. A. Tucker, H. Darmodjo, W. E. Acree, Jr., M. Zander, E. C. 
Meister, M. J. Tanga, and S. Tokita, Appl. Spectrosc. 46, 1630 (1992).

6. H. E. Zimmerman, Quantum Mechanics for Organic Chemists (Academic Press, New York, 1975), pp. 145-146.

7. J. March, Advanced Organic Chemistry: Reactions, Mechanisms and Structure (McGraw-Hill, New York, 1968), pp. 46-48.

8. G.-P. Blümer and M. Zander, Fresenius Z. Anal. Chem. 296, 409 (1979).

9. S. A. Tucker, W. E. Acree, Jr., B. P. Cho, R. G. Harvey, and J. C. Fetzer, Appl. Spectrosc. 45, 1699 (1991).

10. V. L. Amszi, Y. Cordero, B. Smith, S. A. Tucker, W. E. Acree, Jr., C. Yang, E. Abu-Shaqara, and R. G. Harvey, Appl. Spectrosc. 46, 1156 (1992).

11. S. A. Tucker, H. Darmodjo, W. E. Acree, Jr., J. C. Fetzer, and M. Zander, Appl. Spectrosc. 46, 1260 (1992).

12. H. Dreeskamp, E. Koch, and M. Zander, Z. Naturforsch. 30A, 1311 (1975).

13. U. Breymann, H. Dreeskamp, E. Koch, and M. Zander, Chem. Phys. Lett. 59, 68 (1978).

14. S. H. Chen, C. E. Evans, and V. L. McGuffin, Anal. Chim. Acta 246, 65 (1991).

15. J. Iball and W. D. S. Motherwell, Acta Crystallogr., Sect. B 25, 882 (1969).

16. M. L. Bailey, J. P. M. Bailey, and C. A. Coulson, Acta Crystallogr., Sect. B 26, 1622 (1970).
17. M. W. Partridge, S. A. Slorach, and H. J. Vipond, J. Chem. Soc. 3670 (1964).

18. M. W. Partridge and H. J. Vipond, J. Chem. Soc. 632 (1962).

19. D. M. Hall, J. E. Ladbury, M. S. Lesslie, and E. E. Turner, J. Chem. Soc. 3475 (1956).

20. D. G. Bloomfield, C. Upton, and H. J. Vipond, J. Chem. Soc., Perkin Trans. I 857 (1986).

21. C. Upton, J. Chem. Soc., Perkin Trans. I 1225 (1986).

22. S. A. Tucker, V. L. Amszi, and W. E. Acree, Jr., J. Chem. Educ. 69, A8 (1992).

23. C. C. Westcott, pH Measurements (Academic Press, New York, 1978), pp. 112-123.

24. D. A. Skoog, D. M. West, and F. J. Holler, Fundamentals of Analytical Chemistry (Saunders College Publishing, New York, 1992), 6th ed., pp. 430-432.

25. H. A. Laitinen and W. E. Harris, Chemical Analysis (McGrawHill, New York, 1960), pp. 84-88.

26. P. Longhi, T. Mussini, and S. Rondinini, Anal. Chem. 58, 2290 (1986).

27. R. B. Cundall, D. J. W. Grant and N. H. Shulman, J. Chem. Soc., Faraday Trans. 2 78, 737 (1982).

28. J. H. Markgraf and R. J. Katt, J. Org. Chem. 37, 717 (1972).

29. I. J. Pachter and M. C. Kloetzel, J. Amer. Chem. 73, 4958 (1951). 